\title{
Controlling Hyperchaos
}

\author{
Ling Yang,* Zengrong Liu, ${ }^{\dagger}$ and Jian-min Mao* \\ Department of Mathematics, Hong Kong University of Science and Technology, Clear Water Bay, Kowloon, Hong Kong
} (Received 15 March 1999)

\begin{abstract}
For a finite-dimensional dynamical system, whose governing equations may or may not be analytically available, we show how to stabilize an unstable orbit in a neighborhood of a "fully"unstable fixed point (i.e., a fixed point at which all eigenvalues of the Jacobian matrix have modulus greater than unity). Only one of the unstable directions is to be stabilized via time-dependent adjustments of control parameters. The parameter adjustments can be optimized.
\end{abstract}

PACS numbers: $05.45 . \mathrm{Gg}, 05.45 . \mathrm{Pq}$

In many engineering and other practical problems, chaos is undesirable and therefore needs to be controlled. The issue of controlling chaos, however, had not been actively studied until the year of 1990 when Ott, Grebogi, and Yorke (OGY) gave a method for controlling chaos [1]. Experimental works on the feedback stabilization have been carried out $[2,3]$. The method has been extended and modified [4-7], among which the method proposed by Romeiras, Grebogi, Ott, and Dayawansa (RGOD) [4] has attracted much attention. As a related topic, "using chaos to direct trajectories to targets" has also been investigated [8].

From a practical point of view, controlling a "fully" unstable system (i.e., a system having a fixed point with no preexisting stable manifold in its neighborhood) is as interesting and important as the one with a stable manifold. However, the issue of controlling such hyperchaos has not been particularly addressed. Actually, the OGY method [1] is to stabilize an unstable orbit in the neighborhood of a hyperbolic fixed point by forcing the orbit onto the stable manifold. Therefore the method cannot be used to control hyperchaos. The RGOD method [4] uses a feedback matrix which makes the fixed point under consideration fully stable. Therefore, in our opinion, the method is not suitable for controlling hyperchaos since the method changes the stability property of the fixed point completely: it is fully unstable originally but fully stable after the parameter adjustment. In this Letter we use a new idea to stabilize unstable orbits even if there is no preexisting stable manifold nearby.

The systems under our study may or may not be given analytically. In other words, a priori analytical knowledge of system dynamics is not necessary. The unstable manifold can be detected from (chaotic) experimental data by using the embedding procedure (also known as technique of reconstruction) [1,9]. The embedding theorem asserts that if an orbit is in an attractor in the phase space, then the corresponding orbit in the embedding space is also in an attractor (but in the embedding space). The theorem further asserts that the two attractors have the same dimension and the same Lyapunov exponents. Let the unstable orbit to be controlled be in an embedding space of dimension
$N$, where $N \geq 1$ is a finite integer, and be near a fixed point at which the dimension of the unstable manifold is $N_{u}$, where $N_{u} \leq N$ is an integer. $\left(N_{u}=N\right.$ is the case we are particularly interested in.) We formulate the system under consideration by map $T: \boldsymbol{\xi}_{n} \rightarrow \boldsymbol{\xi}_{n+1}$, where

$$
\boldsymbol{\xi}_{n+1}=\mathbf{F}_{\boldsymbol{\epsilon}}\left(\boldsymbol{\xi}_{n}\right) \text {. }
$$

Here $\xi \in R^{N}$ is the dynamical variable, $\boldsymbol{\epsilon} \in R^{N_{u}}$ is a small control parameter vector, and $\mathbf{F}_{\boldsymbol{\epsilon}}\left(\boldsymbol{\xi}_{n}\right)$ is a vectorvalued function of $\boldsymbol{\xi}_{n}$ with $\boldsymbol{\epsilon}$ as parameter. For flows, map (1) is a Poincaré map. We emphasize that this map is formally written (i.e., the map may be given not analytically but given by a data set) and that properties of the vector field $\mathbf{F}_{\boldsymbol{\epsilon}}$ are experimentally accessible.

Let $\boldsymbol{\xi}_{*}^{\mathbf{0}}$ be the fixed point of map (1) with $\boldsymbol{\epsilon}=\mathbf{0}$. We want, by slightly adjusting parameter $\boldsymbol{\epsilon}$, to control an orbit of the map that runs away from the fixed point if $\boldsymbol{\epsilon}=$ $\mathbf{0}$. Let $\mathbf{J}$ be the Jacobian matrix of the map with $\boldsymbol{\epsilon}=\mathbf{0}$ evaluated at the fixed point, i.e.,

$$
\mathbf{J}=\left(\frac{\partial \mathbf{F}_{\mathbf{0}}}{\partial \boldsymbol{\xi}_{n}}\right)_{\boldsymbol{\xi}_{n}=\boldsymbol{\xi}_{*}^{0}} .
$$

The elements of the Jacobian matrix can be determined experimentally in practical problems. Without loss of generality, it is assumed that proper coordinate changes have been made so that $\boldsymbol{\xi}_{*}^{\mathbf{0}}$ is the origin of the $N$-dimensional space and that the eigenvectors of the Jacobian matrix are along the coordinate axes of the space. The eigenvalues of the Jacobian matrix $\mathbf{J}$ in Eq. (2) are all with modulus greater than unity for the case we study in this Letter. Therefore the determinant of the Jacobian matrix is not equal to one, and the implicit function theorem can be used to assert that map (1) with small parameter $\boldsymbol{\epsilon}$ has a fixed point in the neighborhood of $\boldsymbol{\xi}_{*}^{\mathbf{0}}$. Denote this fixed point by $\boldsymbol{\xi}_{*}$ and define the following matrix:

$$
\mathbf{M}=\left(\frac{\partial \boldsymbol{\xi}_{*}}{\partial \boldsymbol{\epsilon}}\right)_{\boldsymbol{\epsilon}=\mathbf{0}} .
$$

Again, the elements of this matrix can be obtained experimentally for a practical problem under study.

When $\boldsymbol{\epsilon}$ is small, consider a neighborhood of $\boldsymbol{\xi}_{*}^{\mathbf{0}}, W$, that is large enough to also include a neighborhood of 
$\boldsymbol{\xi}_{*}$; see Fig. 1 for illustration. We also require $W$ to be large enough so that if $\boldsymbol{\xi}_{n}$ is in $W$ then its image under the mapping, $\boldsymbol{\xi}_{n+1}=\mathbf{F}_{\boldsymbol{\epsilon}}\left(\boldsymbol{\xi}_{n}\right)$, is also in $W$. We write $\boldsymbol{\xi}_{n+1}-\boldsymbol{\xi}_{*} \approx \tilde{\mathbf{J}}\left(\boldsymbol{\xi}_{n}-\boldsymbol{\xi}_{*}\right)$, where $\tilde{\mathbf{J}}$ is the Jacobian matrix of the map with small $\boldsymbol{\epsilon}$ evaluated at $\boldsymbol{\xi}_{*}$. This $\tilde{\mathbf{J}}$ can be approximated by matrix $\mathbf{J}$ given in Eq. (2). Hence, for $\boldsymbol{\epsilon} \rightarrow \mathbf{0}$,

$$
\boldsymbol{\xi}_{n+1}-\boldsymbol{\xi}_{*}=\mathbf{J}\left(\boldsymbol{\xi}_{n}-\boldsymbol{\xi}_{*}\right) .
$$

To stabilize the unstable orbit, we propose to require

$$
\boldsymbol{\xi}_{n+1}=k \boldsymbol{\xi}_{n}
$$

where $k$ is a constant and $-1<k<1$. This means that the orbit is forced to go to the fixed point $\boldsymbol{\xi}_{*}^{0}$ located at the origin. On the other hand, from the definition of matrix $\mathbf{M}$, we have, for $\boldsymbol{\epsilon} \rightarrow \mathbf{0}$,

$$
\xi_{*}=\mathbf{M} \epsilon
$$

When matrices $(\mathbf{J}-\mathbf{I})$ and $\mathbf{M}$ are both invertible, we eliminate $\boldsymbol{\xi}_{*}$ and $\boldsymbol{\xi}_{n+1}$ in Eqs. (4), (5), and (6) to have

$$
\boldsymbol{\epsilon}_{n}=\mathbf{M}^{-1}(\mathbf{J}-\mathbf{I})^{-1}(\mathbf{J}-k \mathbf{I}) \boldsymbol{\xi}_{n},
$$

where $\mathbf{I}$ is the $N \times N$ identity matrix. Here $\boldsymbol{\epsilon}$ has been redenoted by $\boldsymbol{\epsilon}_{n}$ to indicate that the parameter adjustment is in the $n$th iteration of the map. With the parameter adjusted according to Eq. (7), iterations of map (1) give the series $\left\{\boldsymbol{\xi}_{1}, \boldsymbol{\xi}_{2}, \ldots\right\}$ converging to the fixed point $\boldsymbol{\xi}_{*}^{0}$ monotonically, and therefore stabilization is achieved. The constant $k$ in Eq. (7) is arbitrary as long as its absolute value is smaller than unity. The radius of convergence for the control method can be mathematically estimated. For twodimensional systems, we have been able to mathematically estimate the maximum size of the neighborhood $W$ defined

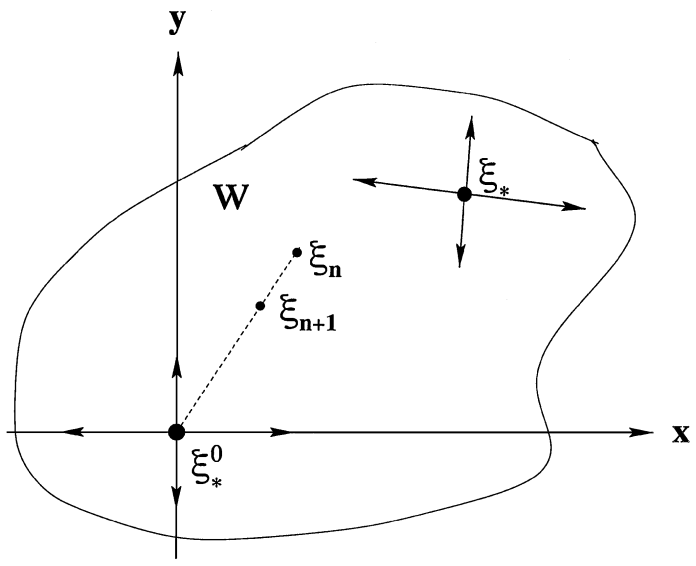

FIG. 1. Schematic illustration of the method of controlling hyperchaos. Point $\xi_{*}^{0}$ is a fully unstable fixed point of map (1), $\xi_{n+1}=F_{\epsilon}\left(\xi_{n}\right)$, when $\epsilon=0$. Point $\xi_{*}$ is the fixed point of the map when $\epsilon$ is nonzero but small. To stabilize an unstable orbit, the parameter $\epsilon$ should be adjusted at each iteration so that $\xi_{n+1}$ is closer to $\xi_{*}^{0}$ than $\xi_{n}$ does along the dashed straight line. previously. This mathematical work will be published elsewhere.

The method of controlling chaos as given by Eq. (7) can be applied to a wide range of problems. It can be used in practical problems, for which a priori analytical knowledge of the system dynamics is usually not available, because the elements of the matrices $\mathbf{J}$ and $\mathbf{M}$ are experimentally accessible. The method can also be used to control a flow by taking a Poincaré map first and then using Eq. (7). Furthermore, the method is formulated for an $N$-dimensional system; there $N$ is a finite integer. Therefore the method can be used for high dimensional problems. When an unstable orbit of period $p$, where $p$ is an integer greater than one, needs to be stabilized, the method can still be used if the $p$-fold composition of the map with itself takes the place of the original map in the formalism.

The idea of the OGY method introduced in Ref. [1] is to force the orbit toward a preexisting stable manifold. Therefore the OGY method cannot be used to control hyperchaos, while our method can because there is no requirement for existence of a stable manifold. Furthermore, the unstable orbit to be controlled is guided toward the stable manifold in the OGY method and, most likely, it falls not exactly on the manifold but on a tiny strip around the manifold. One more iteration of the adjusted map may take the orbit toward an unstable direction where nonlinearity is important, and the orbit continues to wander chaotically as if there were no control. The orbit may eventually return due to ergodicity of the attractor, but only after a long time. This kind of wandering of the orbit may also happen in our method but the odds are much lower because the orbit is forced to go directly towards the fixed point itself (not via the stable manifold). Therefore our method should be more effective in the sense that it takes less time for an unstable orbit under control to reach the fixed point than that in the OGY method.

The idea of the RGOD method [4] is to use the "regulator" matrix $\mathbf{R}$ as the feedback matrix. All eigenvalues of matrix $\mathbf{R}$ are required to have modulus smaller than unity so that, under iterations of the adjusted map $\boldsymbol{\xi}_{n+1}=\mathbf{R} \boldsymbol{\xi}_{n}$, the unstable orbit becomes closer and closer to the fixed point. Therefore the method requires the fixed point to become "fully" stable after the parameter adjustment. In our opinion, it is an over requirement. Our method requires the orbit to be stable along only one direction, the direction connecting $\boldsymbol{\xi}_{n}$ and the fixed point, as shown in Eq. (5). Therefore the parameter adjustment proposed in the RGOD method may change the original system too much, especially if the dimension of the system is high. In addition, the radius of convergence for the RGOD method should be smaller than ours due to the over requirement. Numerical examples are easy to find to show this.

Equation (7) can be rewritten as $\left|\boldsymbol{\epsilon}_{n}\right|=C\left|\boldsymbol{\xi}_{n}\right|$, where $C$ is a constant. In other words, the size of the control perturbation required to implement control is proportional to 
the distance between the fixed point $\boldsymbol{\xi}_{*}^{0}$ and the unstable orbit to be controlled. In either the OGY method or the RGOD method, the control perturbation is also proportional to the distance $\left|\boldsymbol{\xi}_{n}\right|$, as shown in Eq. (2) of Ref. [1] and Eq. (2.6) of Ref. [4]. Considering proportional constant $C$ to be of order one in general, the size of the control perturbation is therefore of the same order as the distance between the fixed point and the unstable orbit to be controlled, no matter if the OGY method, the RGOD method, or our method is used. This is expected because the three control methods are all based on a linearization analysis. The proportional constant ( $C$ in the linear relation $\left|\boldsymbol{\epsilon}_{n}\right|=C\left|\boldsymbol{\xi}_{n}\right|$ ) in our method is given by requiring the system to be stabilized along only one direction, and therefore should be smaller, in general, than the one in the RGOD method that requires the system to be stabilized in all the directions. Furthermore, $k$ in Eq. (7) is a free variable (as long as $|k|<1$ ) and gives an additional device to obtain a smaller control perturbation. (The proportional constant in the OGY method cannot be compared directly with ours because the former involves an eigenvalue of the Jacobian matrix with modulus less than one and the latter does not.)

The control parameter in our method can be adjusted in a range because $k$ in Eq. (7) can be any value between -1 and 1 . Therefore we can optimize parameter adjustment to have a fast control. On the other hand, the number of parameters introduced in either the OGY or the RGOD method is one, less than that in our method. Our method is formulated in a way that the number of control parameters is equal to the dimension of the unstable manifold. However, we can, if needed, make only one of the parameters independent and all other parameters dependent on that parameter in each iteration step. This is done by determining a parameter vector $\boldsymbol{\epsilon}^{\prime}=\mathbf{M} \boldsymbol{\xi}_{n}$ for a given $\boldsymbol{\xi}_{n}$ first, and then letting $\boldsymbol{\epsilon}_{n}=c \boldsymbol{\epsilon}^{\prime}$, where $c$ is a scalar constant. By doing so, only the first, say, element of $\boldsymbol{\epsilon}_{n}$ is independent. We can do so because $\boldsymbol{\xi}_{n}$ is to be dragged to the fixed point along the straight line connecting $\boldsymbol{\xi}_{n}$ and the fixed point on the phase space, and therefore $\boldsymbol{\epsilon}_{n}$ varies also along a straight line, the line connecting $\boldsymbol{\epsilon}_{n}$ and the origin $\boldsymbol{\epsilon}=\mathbf{0}$ in the parameter space.

Finally we give an example to illustrate how hyperchaos can be successfully controlled by adjusting parameters according to Eq. (7). Consider the two-dimensional map [10],

$$
\left\{\begin{array}{l}
x_{n+1}=1-2\left(x_{n}^{2}+y_{n}^{2}\right)+p \\
y_{n+1}=-4 x_{n} y_{n}+q
\end{array}\right.
$$

This map is in form (1) if we let $\boldsymbol{\xi}=(x, y)$ and $\boldsymbol{\epsilon}=(p, q)$. The map has four fixed points, which can be analytically written down. Here we consider only one of them, the one with coordinates $x_{*}=\frac{1}{8}[-2+\sqrt{1+8(1+p+q)}+$ $\sqrt{1+8(1+p-q)}]$ and $y_{*}=\frac{1}{8}[\sqrt{1+8(1+p+q)}-$

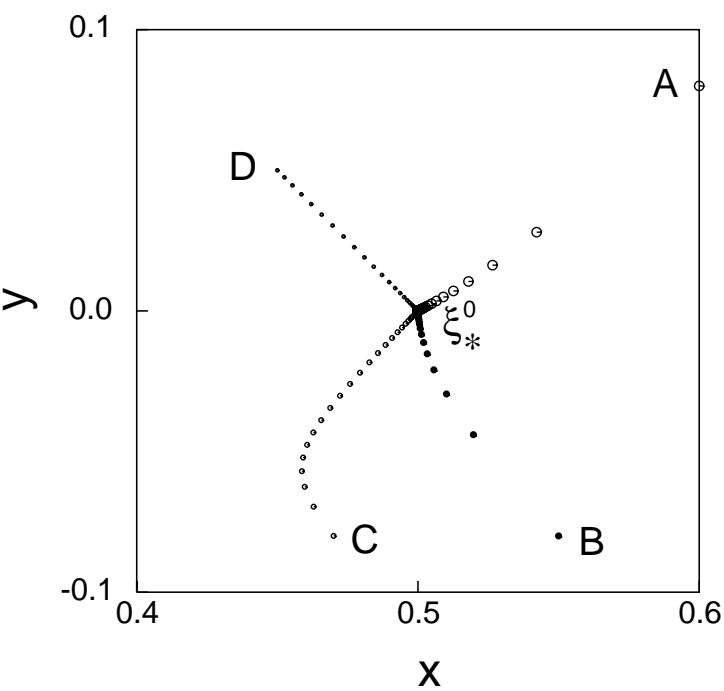

FIG. 2. Numerical results for map (8) in the example. Four orbits are shown, starting at points $A, B, C$, and $D$, respectively. All of them converge to the fully unstable fixed point $\xi_{*}^{0}=\left(\frac{1}{2}, 0\right)$ very quickly under iterations of the map with parameters $p_{n}$ and $q_{n}$ given by Eq. (7).

$\sqrt{1+8(1+p-q)}]$. When $p=q=0$, the fixed point is at $\boldsymbol{\xi}_{*}^{\mathbf{0}}=\left(\frac{1}{2}, 0\right)$. It is easy to see that $\mathbf{J}=\left(\begin{array}{cc}-2 & 0 \\ 0 & -2\end{array}\right)$ and $\mathbf{M}=\left(\begin{array}{cc}1 / 3 & 0 \\ 0 & 1 / 3\end{array}\right)$. Therefore the parameters $p_{n}$ and $q_{n}$ in the $n$th iteration should be adjusted to $p_{n}=(2+$ $k)\left(x_{n}-\frac{1}{2}\right)$ and $q_{n}=(2+k) y_{n}$. Figure 2 shows results of our numerical computation when $k=\frac{3}{4}$. In the figure, there are four orbits starting from points $A, B, C$, and $D$, respectively. Under iterations of map (8) with the adjusted parameters, the four orbits all converge to the fully unstable fixed point at $\left(\frac{1}{2}, 0\right)$ monotonically and very fast.

In conclusion, we have shown how an unstable orbit in the neighborhood of a fully unstable fixed point can be stabilized. The stabilization is done by adjusting the control parameter so that one of the unstable directions becomes stable. The previously known OGY method [1] cannot be used to control such hyperchaos because the method requires preexistence of stable manifold. The RGOD method [4] also cannot be used to control hyperchaos because the method requires all unstable directions to become stable after parameter adjustment and therefore changes the intrinsic stability property of the original system completely. Our method of controlling hyperchaos can be used not only for a system analytically given, but also for a system in a practical situation where no priori analytical knowledge of the system dynamics is available. The method can be applied to any finite-dimensional system, including mapping and flow.

This work is partially supported by the Hong Kong Research Grant Council Grant No. HKUST606/95P and by the National Natural Science Foundation of China. 
*Permanent address: LNM, Institute of Mechanics, Chinese Academy of Sciences, Beijing 100080, China; and Department of Mathematics, Suzhou University, Jiangsu, Suzhou 215006, China.

†Permanent address: LNM, Institute of Mechanics, Chinese Academy of Sciences, Beijing 100080, China; and Department of Mathematics, Shanghai University, Shanghai 201800, China.

† To whom correspondence should be addressed.

Email address: mamao@uxmail.ust.hk

[1] E. Ott, C. Grebogi, and J. A. Yorke, Phys. Rev. Lett. 64, 1196 (1990).

[2] W. L. Ditto, S. N. Rauseo, and M. L. Spano, Phys. Rev. Lett. 65, 3211 (1990).

[3] J. Singer, Y-Z. Wang, and H. H. Bau, Phys. Rev. Lett. 66, 1123 (1991).
[4] F. J. Romeiras, C. Grebogi, E. Ott, and W. P. Dayawansa, Physica (Amsterdam) 58D, 165 (1992).

[5] D. Auerbach, C. Grebogi, E. Ott, and J. A Yorke, Phys. Rev. Lett. 69, 3479 (1992).

[6] K. Pyragas, Phys. Lett. A 170, 421 (1992).

[7] V. Petrov, B. Peng, and K. Showalter, J. Phys. Chem. 96, 7506 (1992).

[8] T. Shinbrot, E. Ott, C. Grebogi, and J. A. Yorke, Phys. Rev. Lett. 65, 3215 (1990); Phys. Rev. A 45, 4165 (1992); T. Shinbrot, W. Ditto, C. Grebogi, E. Ott, M. Spano, and J. A. Yorke, Phys. Rev. Lett. 68, 2863 (1992).

[9] P. So, E. Ott, S. J. Schiff, D. T. Kaplan, T. Sauer, and C. Grebogi, Phys. Rev. Lett. 76, 4705 (1996).

[10] K. Kaneko, Physica (Amsterdam) 34D, 1 (1989). 